\title{
Positive behaviour support in frontotemporal dementia: a pilot study
}

Authors: Claire M O’Connor ${ }^{1,2,3}$, Eneida Mioshi ${ }^{4}$, Cassandra Kaizik ${ }^{2,5}$, Alinka Fisher $^{6}$, Michael Hornberger ${ }^{4,7}$, Olivier Piguet ${ }^{2,7,8}$

\section{Affiliations:}

${ }^{1}$ HammondCare, Centre for Positive Ageing, Sydney, Australia,

${ }^{2}$ The University of Sydney, Brain and Mind Centre, Sydney, Australia

${ }^{3}$ The University of New South Wales, Sydney, Australia

${ }^{4}$ University of East Anglia, Faculty of Medicine and Health Sciences, UK

${ }^{5}$ The University of Sydney, Sydney Medical School, Sydney, Australia

${ }^{6}$ Flinders University, College of Nursing and Health Sciences Adelaide, Australia

${ }^{7}$ ARC Centre of Excellence in Cognition and its Disorders, Sydney, Australia

${ }^{8}$ The University of Sydney, School of Psychology, Sydney, Australia

Corresponding author: Dr Claire M O'Connor

HammondCare

Centre for Positive Ageing

4 Spicer Avenue, Hammondville, NSW, Australia, 2170

Phone: +6128788 3948

Email: coconnor@hammond.com.au

ORCiD: https://orcid.org/0000-0002-3541-708X

Twitter:@ClaireMOConnor1

Word count: 5475 


\section{Abstract:}

Frontotemporal dementia (FTD) is a progressive neurodegenerative brain condition clinically characterised by marked changes in behaviour that impact the individuals' relationships and community participation, and present challenges for families. Family carers of individuals with FTD find apathy and disinhibition particularly challenging leading to high levels of stress and burden. Positive behaviour support (PBS) as a behaviour intervention framework has never been trialled in FTD. This pilot study examined the functional basis of apathetic and disinhibited behaviours in four FTD dyads and explored the acceptability of a PBS intervention. The PBS program was provided by an occupational therapist in the participants' homes. Measures collected at baseline and post intervention (M=3.9 months) assessed: function of behaviours, challenging behaviours, and qualitative outcomes pertaining to acceptability of the PBS approach. PBS was an acceptable intervention for all four dyads. 'Sensory' and 'tangible' were the most common functions contributing to maintenance of behaviour changes, and aspects of apathetic and disinhibited behaviours improved following intervention. This study demonstrates the acceptability and potential benefit of a PBS program to provide support in FTD. A more rigorous trial will be an important next step in developing improved services tailored to the needs of this unique population.

Keywords: Positive behaviour support, frontotemporal dementia, challenging behaviour, family support, family carer 


\section{Introduction:}

Frontotemporal dementia (FTD) is a younger-onset dementia (onset $<65 \mathrm{yrs}$ ), associated with changes in behaviour and/or language that present challenges for families and support staff, and impact the individuals' relationships and community participation. Behavioural-variant FTD (bvFTD) and semantic dementia (SD) are the two subtypes of FTD where challenging behaviours most commonly occur (Hodges, 2001; O'Connor et al., 2016; Rosen et al., 2006). These behaviours are those that may be difficult to manage, impact negatively on the person or their environment, and often result in high levels of burden in carers or family members (Andrews, 2006; Mioshi et al., 2013). Common changes observed in FTD include disinhibition, apathy, changed eating behaviours, loss of insight, compulsive behaviour, and impulsivity (Shinagawa, 2013; Snowden et al., 2001), some of which are subtype-specific. For instance, changes in eating behaviours differ in bvFTD and SD, where individuals with bvFTD may be likely to experience gluttony and be indiscriminate around food, while individuals with SD may be more likely to demonstrate specific and rigid daily routines around food (Snowden et al., 2001). Nevertheless, individuals with SD and bvFTD share many behaviour changes, such as disinhibition and stereotypical behaviours (O’Connor et al. 2016).

Behaviour changes in FTD result from a complex interplay of variables such as brain atrophy, cognitive changes (e.g., lack of insight, decreased comprehension of social norms), communication impairments, and environmental stimuli (Ghosh et al. 2013; Kortte \& Rogalski, 2013; Marczinski et al., 2004; Massimo et al., 2009). Understanding how these variables interact is vital for the development of effective interventions. 
Apathy and disinhibition in particular are often cited as being the most challenging behaviours for family members (Massimo et al., 2009; Whitwell et al., 2009). Management of such behaviours has been attempted through off-label medications, yet results have been disappointing, often with unanticipated side effects (Haass \& Neumann, 2016; Tsai \& Boxer, 2014; Tsai \& Boxer, 2016), and diseasemodifying treatments are still lacking in FTD. Effective non-pharmacological approaches that explore the use of compensatory methods to address the environmental, cognitive and sensory basis of behaviours are therefore needed (Kortte \& Rogalski, 2013), but remain limited (O'Connor et al., 2013). A recent pilot study demonstrated the potential for using an activity-based intervention to maintain everyday function and reduce behavioural symptoms overall in a cohort of individuals with FTD (O’Connor et al. 2017a), supporting the need to further develop such psychosocial intervention approaches.

Apathy and disinhibition are common in FTD and form a central and challenging aspect of supporting a person with FTD (O'Connor et al., 2016; O'Connor et al., 2017b). As such, effective behavioural interventions are of the utmost importance. Positive behaviour support (PBS) is one intervention approach that has been used successfully in supporting adults with intellectual disability and brain injury (Hassiotis et al., 2009; Feeny \& Achilich, 2014), but is yet to be trialled in FTD. Effective PBS involves a comprehensive functional behaviour assessment and analysis, which then inform person-centred interventions that increase quality of life and reduce the occurrence of behaviours (Allen, James, Evans, Hawkins, \& Jenkins, 2005; Carr et al., 2002; Horner et al., 1990). Crucially, PBS has been found to be an efficacious intervention approach for people with severe and challenging behaviours (LaVigna \& Willis, 2012), and a similar approach has been trialled in general 
dementia cohorts with encouraging results (Bird et al. 2007; Bird et al. 2009). While these studies primarily involved people with undifferentiated dementia diagnoses living in residential care with care staff (Bird et al. 2007), the latter study included a small cohort of community-dwelling individuals and their family carers (Bird et al. 2009). This highlights the potential of PBS for addressing the common challenging behaviours of apathy and disinhibition seen in FTD.

The underlying assumption of PBS is that challenging behaviours serve a function (purpose) for the individual. A functional behaviour assessment and analysis is conducted in order to identify the factors underlying challenging behaviours, and determine the function/s they serve for the individual. This assessment then informs a multi-component PBS plan, which includes a range of strategies from within four key domains: 1) ecological strategies (addressing any mismatch between the person and their environment; 2) positive programming (teaching skills that are functionally related/equivalent, and coping strategies; 3 ) focused support strategies (controlling antecedents and reinforcing desired behaviours; and 4) reactive strategies (reducing the impact of a behaviour when it is occurring) (LaVigna \& Willis, 2005, 2012; McLean \& Grey, 2012).

In general dementia care facilities (housing predominantly older individuals with Alzheimer's disease and/or vascular dementia), behaviour changes can arise as a result of the dementia-related brain changes or of unmet needs, such as pain or loneliness (Algase et al., 1996; Cohen-Mansfield, 2000), in addition to the progression of the disease. In this context, behaviour support largely becomes about recognising and addressing these unmet needs. In FTD, however, behaviour changes have a primary biological basis arising from the location and severity of brain atrophy found in the frontal and temporal lobes in this population (Massimo et al. 2015; 
Williams, Nestor, \& Hodges, 2005; Zamboni et al. 2008). Indeed, these brain regions are known to play a central role in modulating behaviours (Hodges \& Piguet, 2018). While behavioural symptoms in FTD may in part also reflect unmet needs and reactivity to environmental stimuli (Kortte \& Rogalski, 2013), effective strategies to support behaviour changes in FTD are likely to differ from those used in general dementia populations. The PBS framework, which assumes that the behaviour has a function (i.e., serves a purpose) for the individual, has not yet been explored in detail in FTD. It may help determine the variables underlying challenging behaviours (e.g., through functional assessment and analysis) to inform targeted intervention plans.

Individuals with FTD living in the community often live with a family member (providing support), and research highlights the importance of involving these family members in behavioural interventions (Martini de Oliveira et al., 2019; O'Connor et al., 2013). A recent pilot study demonstrated the feasibility of working closely with a family member of an individual with FTD while implementing a community-based activity intervention to address behavioural changes such as apathy, disinhibition and agitation (O'Connor et al., 2017a). PBS may therefore be an appropriate intervention in this population, allowing for a similar format of working closely with both the individual and their family to address challenging behaviours.

Given the often severe and challenging nature of behaviour changes in bvFTD and SD, this study sought to a) understand the functional basis of apathetic and/or disinhibited behaviours identified as challenging by the person's family member, and explore the impact of PBS on these behaviours, and b) consider the acceptability of a PBS intervention for individuals with FTD and their family support person (dyads) living in the community. We hypothesised that as family members implemented PBS plans targeting the identified functional basis of behaviour, levels of apathetic and 
disinhibited behaviours would improve. We further anticipated that PBS would be an acceptable intervention for families, but behavioural improvements would not necessarily translate to improved everyday function.

\section{Methods:}

\section{Participants}

Participants were recruited through FRONTIER, a clinical research group based at the Brain and Mind Centre of the University of Sydney, Australia that investigates frontotemporal dementia and related younger-onset dementias. Information about the study was mailed out to potential participants $(\mathrm{n}=21)$ and 16 $(76 \%)$ were returned. Potential candidates were identified if they met the following inclusion criteria: diagnosis of either possible or probable bvFTD or SD (GornoTempini et al., 2011; Rascovsky et al., 2011) by a multidisciplinary consensus involving neurologists and neuropsychologists; presence of behaviour changes over the past month as measured by the revised Neuropsychiatric Inventory Clinician scale (NPI-C) apathy and disinhibition items; were living in the community with a family support person willing to participate in the intervention; and absence of a major depression (assessed via case notes and multidisciplinary consensus). Disease duration at the time of baseline assessment was estimated from the date of symptom onset as described by the family member. Due to time constraints, four individuals with FTD (two with bvFTD and two with SD) and their family support persons were selected at random from among the pool of potential participants. In the SD participants, one showed predominantly right-sided brain atrophy (SD-right) and the other had predominantly left-sided brain atrophy (SD-left) on MRI. 
The study was approved by the South Eastern Sydney Local Health District and the University of New South Wales ethics committees. Each participant and/or family member (i.e., person responsible) where appropriate, provided written informed consent to participate in the research. Capacity for the individual to provide their own consent was determined via patient notes and in consultation with their neurologist. In the cases where participants with dementia were deemed not to have sufficient capacity to provide consent, they were still supported to make a decision regarding their participation using the tenets of supported decision making (Sinclair et al. 2018).

\section{$\underline{\text { Study design }}$}

Using a pre-test post-test design, baseline assessments were collected by an occupational therapist $(\mathrm{CMOC})$ and were repeated post-intervention $(\mathrm{M}=3.9$ months, range 2.7 - 5.1 months) by a second occupational therapist (CK) not involved in implementing the PBS interventions. Assessments involved a combination of qualitative and quantitative measures to evaluate program acceptability, everyday living function (Disability Assessment for Dementia; Gelinas, Gauthier, McIntyre, \& Gauthier, 1999) and behavioural symptoms (Neuropsychiatric Inventory - Clinician rating scale; de Medeiros et al., 2010).

\section{$\underline{\text { Intervention: Positive Behaviour Support }}$}

An occupational therapist (CMOC) experienced in implementing psychosocial interventions with people with dementia, and trained in the PBS approach worked with each dyad in their home to develop and implement a tailored behavioural support plan. The PBS intervention protocol had two assessment components: a 
comprehensive functional assessment of the behaviour/s, and observations of the patient within their home environment. The comprehensive assessment of behaviour/s involved a review of the clinical notes, and an in-depth semi-structured interview process with the family support person using a Functional Behavioural Assessment interview form (O’Neill et al., 1997) and the Motivational Assessment Scale (Durand \& Crimmins, 1988). Direct observation over the initial two visits focused both on the challenging behaviours as well as the positive interactions within the dyad. The family member was trained in using an $\mathrm{ABC}$ (Antecedent-Behaviour-Consequence) chart, which they used to record behavioural incidents between each of the therapist's visits. This multi-factor assessment process provided an efficient approach to data collection, which was used to identify factors influencing the occurrence of the challenging behaviour (e.g., physical and social environment) and inform a functional hypothesis. A data-based PBS plan was then developed to be implemented with the person with dementia and their family member with support from the therapist. Plans were individualised based on identified behaviours and functional hypotheses, with a focus on environmental and preventative strategies. In each case, these strategies were based around positive reinforcement of desired behaviours, 'extinction' (purposefully not reinforcing undesired behaviour) in the instances where this was deemed appropriate, and teaching acceptable replacement (i.e., functionally equivalent) behaviours (Fisher et al., 2019). As challenging behaviours in FTD have a primarily biological cause, extinction-based techniques were only applied in specific instances and with careful planning. For example, it was deemed appropriate and safe to ignore swearing behaviour with $\mathrm{P} 1$, with positive reinforcement used at the same time to increase desired behaviours serving the same purpose (i.e. those more efficient and 
effective in communicating their needs) Appendix 1 provides an overview of the behaviour support plans developed for each participant.

The PBS intervention was completed over a period of three months $(\mathrm{M}=3.2$ months, range 2.1 - 4.7 months) and implemented over five to seven in-home visits (session length $\mathrm{M}=80$ mins, range 45 - 160 mins) depending on the needs of each dyad e.g., more severe challenging behaviours (P1), or a family support person who identified less need for ongoing support (P3). The first two visits were planned to be completed over two weeks; remaining visits were planned more flexibly to accommodate participant schedules, and occurred on average every two to three weeks $(\mathrm{M}=2.8$ weeks, range $2.3-3.4$ weeks $)$. The family member was asked to select three behaviours of an apathetic or disinhibited nature that were particularly challenging to be the focus of intervention. Visits one and two involved functional assessment of these behaviours, and at the end of this period a PBS plan for each of the three behaviours was developed in collaboration with the family member (and the person with dementia where appropriate). Each protocol consisted of three behaviours, each with specific strategies included in the PBS plan. The individual sessions with the therapist also provided each dyad with opportunities for education and skill development regarding the implementation of PBS strategies, with this approach recommended to effectively include family members as active members of the team (Fisher et al. 2017). The remaining visits focused on implementation of the PBS plans in a staggered approach, allowing the family member time to practice strategies between sessions. At each successive visit progress was monitored and any issues problem-solved with the therapist and family member before the next PBS plan was introduced. 


\section{$\underline{\text { Measures }}$}

Baseline dementia stage was measured using the Frontotemporal dementia Rating Scale (FRS) (Mioshi et al. 2010). The FRS assesses a combination of everyday living skills and behaviour changes across 30 items. The raw score is converted to a Rasch score which is indicative of disease severity progression from "very mild" to "profound".

The functional behaviour assessment completed during the PBS process involves the use of different assessment tools as described above. To understand the intervention process for each participant in the current study, results from one of these tools, the Motivational Assessment Scale will be presented alongside the measures used to assess program acceptability and efficacy.

\section{PBS intervention acceptability (preliminary data)}

During the intervention period, implementation of the prescribed PBS plans was carefully documented by the therapist, using structured notes. Whether the family member had attempted to implement the plan between visits was noted, along with information relating to any challenges or positives that were experienced when implementing the strategies with the person with dementia. Common in bvFTD, lack of insight may also occur in SD (Hornberger et al. 2014; Mendez \& Shapira, 2011; Savage et al. 2015); as such, we measured the acceptability of the program by the person with dementia via proxy. This proxy measurement involved therapist notes based on observation and in-depth discussion with the family member on how the person with dementia was perceived to receive the intervention. Upon completion of the program, family members were qualitatively asked by the independent occupational therapist (CK) about their experience with the program. Specifically, the 
interview involved a combination of closed (whether they perceived any benefit in the program and whether they would recommend the program to other families living with FTD) and open-ended questions (what did they find most beneficial about the program or what did they feel was lacking, and what would they change about the program). Participants were also provided the opportunity to contribute any other comments regarding their involvement in the program.

\section{Understanding the function of behaviours}

The Motivational Assessment Scale (MAS; Durand \& Crimmins, 1988) measures the functions the target behaviours may serve for a person. The MAS comprises 16 questions, each pertaining to one of four behavioural function subscales: Tangible (the behaviour relates to something physical the person wants), Escape (the behaviour relates to something the person is trying to avoid, such as a difficult or unwanted task/situation), Attention (the behaviour relates to the person wanting to be noticed or to have more attention paid to them), and Sensory (the behaviour relates to something in the person's environment that they are reacting to as the associated sensation either feels good or bad). Each question is rated by the family support person on a scale ranging between 0 ("never") to 6 ("always"), with scores tallied and mean ratings calculated for each subscale. Higher scores are indicative that that function may be contributing to the maintenance of the target behaviour.

\section{Behaviours}

The NPI-C was used to measure presence of apathetic and disinhibited behaviours broadly, that is, beyond the three specified target behaviours (de Medeiros et al., 2010). This approach was selected for two reasons. First, apathy and 
disinhibition vary across individuals. As such, the aim was to measure the broad impact of the intervention to facilitate generalisability. Second, previous studies have suggested that psychosocial interventions may have a more generalised impact on behavioural changes (Gitlin et al. 2008; O’Connor et al. 2017a). Family members were asked all follow-up questions for the NPI-C Domains of Apathy and Disinhibition to get a rating of frequency, ranging from 0 ("never") to 4 ("very frequently, once or more a day"), as well as severity/intensity, ranging from 0 (“none") to 3 ("'marked, a major source of behavioural abnormality”), and 'carer' distress, ranging from 0 ("not distressing) to 5 ("extremely"). The use of specific domains from the NPI-C has been applied previously (de Medeiros et al., 2010; Gitlin et al., 2016).

\section{Everyday function}

The individual's level of functioning in everyday activities was measured using the Disability Assessment for Dementia (DAD; Gelinas et al., 1999), in order to measure any secondary impacts of the intervention beyond behavioural changes. The DAD measures everyday functioning across 40 items, 17 focusing on basic activities of daily living (ADLs), and 23 on more complex instrumental ADLs. Basic ADLs involve tasks such as eating, toileting and dressing, while instrumental ADLs involve tasks such as organising the finances, shopping or cooking a meal (Lawton \& Brody, 1969; Trombly, 1993). The total DAD score is reported as a percent score, with nonapplicable items excluded (e.g. if the person never did any cooking). Higher scores are associated with better functional abilities.

\section{$\underline{\text { Data analysis }}$}


MAS items were scored as per the protocol described above (Durand \& Crimmins, 1988; Ray-Subramanian, 2013). Each subscale was then ranked from $1-4$ to identify the variables most likely contributing to each behaviour, with 1 indicating the most likely contributor. Scores were tallied to determine the primary (i.e., function with the most scores of 1) and secondary (i.e., function with the most scores of 2) function of behaviours, and were ranked from $1-4$ in the same way as each subscale. Data for the NPI-C and DAD items were analysed using percentage change scores from baseline to post intervention. Percentage change was calculated for each individual case by dividing the change score (baseline score subtracted from the post intervention score) by the absolute value of the baseline score, multiplied by 100 . The individual case $(n=4)$ percentage change scores were then pooled to generate a mean percentage change score for each item across the cohort. These scores were supported with the range of raw individual percentage change scores. Using percentage change enables for individual differences between participants in baseline scores across behavioural and functional items to be compared, and has been used previously in small sample studies (Savage, Piguet, \& Hodges, 2014). Data pertaining to specific participants is identified via participant number (e.g., P1) to facilitate crossreferencing with the tables and appendices.

\section{Results:}

All dyads were living together and in spousal relationships. Demographic and clinical characteristics are shown in Table 1.

Insert table 1 about here 


\section{$\underline{\text { Preliminary results on program acceptability }}$}

Therapist notes indicated that all family members implemented at least one of the three behavioural support plans that were developed during the intervention process. Two family members (P1 and $\mathrm{P} 4)$ implemented all three of their plans and one implemented two plans (P2) but was planning to implement the third upon returning from a trip overseas. The fourth family member (P3) only implemented strategies specific to one of the identified behaviours. This family member had reported considerable stress regarding his wife's behaviour changes. He was not able to provide specific examples about applying the other two plans, commenting that he felt that they had been "using the strategies in general". This dyad was also absent for an extended period of time, limiting their engagement with the PBS intervention.

Family members demonstrated enhanced skills in the face of challenging behaviours. For example, one person (P2) reported on trying to remain calm when her husband became agitated, while another (P1) recognised the benefits of engaging her husband in appropriate behaviours (e.g., gardening) to distract him from shoplifting. In general, individuals with dementia responded well to the strategies. In one instance, the person (P1) began storing his shoplifted trinkets throughout the house, which became too much for his wife to handle. Although he was initially angry when his wife moved the items to one area in the home, this became an acceptable solution to both members of the dyad. In another case (P4), the carer was able to prompt her husband with apathetic behaviour to fix their screen door by setting the activity up for him, providing careful encouragement and framing the activity so he felt in control.

Overall, PBS was an acceptable intervention for all dyads $(n=4)$. All family members felt they received a benefit from the program, making comments such as: " $I$ can refer back to things that Claire has taught me... Gave me ideas about how to 
manage. I have more insight into the condition" (P1). All family members further indicated that they would recommend the program to other FTD families. Three family members commented they would not change anything about the program, while one commented she wished for "more examples of how else to manage things" (P4).

Insert table 2 about here

\section{$\underline{\text { Function of behaviours }}$}

As instructed, the majority of identified behaviours were related to apathy or disinhibition (e.g., decreased communication/social engagement, shoplifting, gambling). In a few instances, other challenging behaviours were also identified as a priority by the family member (e.g., anxiety, changing plans at the last minute). The MAS was completed across the three identified behaviours for each participant, revealing that, overall, the most common primary motivation behind behaviours was 'sensory' followed by 'tangible' (Table 2). The most common secondary overall behavioural motivations were 'escape' followed by 'tangible'. Looking at apathetic behaviours, the primary motivation was 'sensory', followed by 'escape'. The primary motivation for disinhibited behaviours was 'sensory', followed by 'tangible'. Attention was the least likely motivation behind the behaviours expressed by these participants with FTD.

\section{$\underline{\text { Behaviour }}$}

Changes in apathy and disinhibition for each participant are plotted on figures 1a and $1 \mathrm{~b}$. Taken together, levels of apathetic and disinhibited behaviours changed 
between baseline and post intervention. Although the frequency of apathy worsened overall across participants $(M=+21 \%$, range -40 to $72 \%)$, its severity $(M=-12 \%$, range 5 to $-45 \%)$ and associated carer distress $(M=-23 \%$, range -23 to $-43 \%)$ both declined. Visual inspection of the individual participant graphs illustrates a heterogeneous pattern of apathy across both diagnoses; P3 who had two out of three target behaviours relating to apathy demonstrated an increase in severity (Figure 1a). This dyad only introduced strategies specific to one of the identified behaviours in their plan.

Disinhibited behaviours improved across participants on all measures, with lower frequency $(M=-29 \%$, range 4 to $-58 \%)$, severity $(M=-34 \%$, range -19 to $53 \%)$, and associated carer distress $(\mathrm{M}=-27 \%$, range 14 to $-57 \%)$ following interventions. Visual inspection suggests a similar effect across both SD and bvFTD diagnoses. P2 demonstrated no change (or even a slight increase) in disinhibition severity and a slight increase in carer distress (Figure 1b). In this dyad, gambling (and associated financial issues) was a challenge that persisted beyond the intervention period and may have impacted on carer distress.

Insert figure $1 \mathrm{a}$ and $\mathrm{b}$ about here

\section{Everyday functioning of participants}

All individuals with FTD declined in their everyday functional abilities from baseline to post intervention, as expected (Total DAD: $\mathrm{M}=-28 \%$, range -12 to $-42 \%$ ). The largest decline was in instrumental ADLs $(M=-44 \%$, range -33 to $-65 \%)$, with smaller decline seen in basic ADLs $(\mathrm{M}=-12 \%$, range 7 to $-43 \%)$. 


\section{Discussion}

This study is the first investigation of the use of a PBS intervention to address challenging behaviours in individuals with FTD. These results suggest the acceptability and benefit of a PBS program for this population.

Potential for reductions in the severity of apathetic and disinhibited behaviours is encouraging, given that these clinical features are pervasive in FTD and often difficult to manage (Massimo et al., 2009; O'Connor et al., 2016; Whitwell et al., 2009). The behavioural goals selected by carers in this study differed according to FTD subtype. The bvFTD dyads focused on apathetic or other behaviours, while the focus was largely on disinhibited behaviours for SD dyads. This adds to previous work identifying that specific behaviour changes may be more pronounced according to FTD subtype (O’Connor et al. 2016), and suggests that this influences the challenges perceived by family carers.

Impact of the intervention on apathetic behaviours varied across cases. Although the frequency of apathy episodes reported by the family member worsened in three out of four cases (as seen in other studies, O'Connor et al., 2016), this was not mirrored by an increase in the level of severity, or by carer distress. This finding provides support for the PBS approach for both the individual and their family member. Apathy is a chronic clinical feature in FTD that tends to worsen with disease progression (O'Connor et al., 2016). As such, the potential for reduction in severity of apathy is an exciting finding that deserves further research in larger trials of PBS. Interestingly, two participants (P1 and P3) showed a slight worsening of apathy severity, indicating that not all individuals with FTD may benefit from the PBS approach. It is important to note, however, that P3 engaged in only one of the PBS plans throughout the intervention period. Nevertheless, family member distress 
regarding apathy improved in all cases, even in the presence of the increase in apathy frequency in P3. This finding demonstrates that the benefits of PBS extend beyond the individuals with dementia and also has a positive impact on the family by helping them develop better management or coping abilities.

Overall reduction in disinhibited behaviours was found across all dimensions (frequency, severity, and family member distress) and both in individuals diagnosed with bvFTD and SD, as supported by the visual inspection of individual participant graphs. This is a potentially important finding as it remains unclear how these behaviours evolve with disease progression in these dementia subtypes as a whole. One previous study reported improving levels in bvFTD but a worsening in SD with disease progression (O'Connor et al., 2016), whereas another study reported a worsening in both groups over time (Van Langenhove, Leyton, Piguet, \& Hodges, 2016). This study suggests that PBS has potential to result in a reduction in the presentation of disinhibited behaviours in individuals diagnosed with bvFTD or SD. While our findings are encouraging, the benefits of PBS to improve disinhibition in these populations will need to be replicated in larger samples.

Importantly, the MAS assessment helped shed light on family members' perceptions of why individuals with FTD engage in challenging behaviours. In this group, target behaviours most commonly served a 'sensory' function, which suggests these behaviours are pleasurable (self-reinforcing) for the individuals. For example, lying on the couch and doing less around the house, may give the person feelings of pleasure by relaxing and not engaging in physical or cognitive activities. 'Tangible' was the second most common function overall (and specifically for disinhibited behaviours), indicating that performing a behaviour will result in a gain; for example, an individual may swear when told they cannot have something, however, this 
swearing then results in gaining access to the desired item. The 'escape' function was the most common secondary motivation (and the second primary function for apathetic behaviours), suggesting that performing the behaviour assists the person to avoid a situation or task they do not want to do (Durand \& Crimmins, 1988; Singh et al., 1993). Interestingly, 'attention' was rarely associated with the function of behavioural symptoms in this group. This may be related to the common social withdrawal reported in the literature, where individuals with FTD become increasingly less inclined to participate in social situations and seek to avoid busy settings (Shinagawa, Ikeda, Fukuhara, \& Tanabe, 2006; Snowden et al., 2001). Understanding the function of behaviours could have important implications for the development of effective intervention approaches. Challenging behaviours most commonly served a sensory function in this cohort, which suggests that people with FTD may be reactive to their environment (Durand \& Crimmins, 1988). This has important implications for practitioners developing behaviour support plans (see Appendix 1).

Acceptability of this PBS intervention was demonstrated by engagement from the person with dementia in the PBS strategies implemented by family members, in addition to the positive qualitative feedback provided by the family members regarding their involvement in the program. The fact that half of the family members implemented all three PBS plans with a third family member actively planning to implement their third plan is an important positive aspect to consider when investigating potential intervention approaches for individuals with FTD. As FTD is a predominantly younger-onset dementia, individuals with the condition and their families are often facing a range of concurrent challenges, such a juggling employment or caring for dependent children (Armari, Jarmolowicz, \& Panegyres, 
2013; Kaiser \& Panegyres, 2007). The PBS approach allows for the therapist to work with each family within their own personal situation to build capacity in these important support people. The uptake and implementation by the families of the behavioural support plans in this study therefore indicates the potential suitability for PBS as an intervention approach for this unique FTD cohort.

In line with the expected trajectory of ADL functioning seen in people with dementia, all areas of ADLs measured in this study declined from baseline to followup. A smaller decline was observed for basic ADLs than for instrumental ADLs, which aligns with the commonly reported pattern of functional decline for individuals with FTD (Mioshi \& Hodges, 2009; Wicklund, Johnson, Rademaker, Weitner, \& Weintraub, 2007). This finding was somewhat surprising, given the positive impact previously reported of such behavioural interventions on everyday functioning (O’Connor et al. 2017a; Staal et al. 2007).

Although findings are promising, results should also be taken with caution given the small sample and research design. The basic pre-post design limits the conclusions that can be drawn about intervention effect, however, this was a pilot study, with preliminary results warranting studies with more rigorous methodologies. For instance, it is possible that psychosocial interventions other than PBS may result in similar outcomes. In order to determine a clearer link between PBS and positive behaviour outcomes, future studies should implement more robust single-case experimental design methodologies, such as applying multiple baselines across different behaviours (Kazdin, 2016; Tate et al. 2014). Given the known differences in behaviour between bvFTD and SD (O'Connor et al., 2016; Snowden et al., 2001), including them as a mixed cohort is a potential limitation that will need addressing in larger trials as the results may differ between these subtypes. Ongoing work will also 
need to consider including a control group to account for the possible contribution of disease progression rather than as a result of the intervention. While this option may not always be possible (as in this study setting), alternative methodologies such as applying ongoing assessment prior to and during the intervention period should be considered (Kazdin, 2016). Measuring broad apathetic and disinhibited behaviour change via the NPI-C rather than measuring change to the specific target behaviours limits interpretability of the direct impact of the PBS intervention for each case. Future studies should measure specific target behavioural changes, as well as measuring a wider range of behavioural symptoms, i.e., including all NPI-C domains. Finally, assessment of acceptability of this approach was limited by using only therapist session notes and interview data. Future work will benefit from a formal analysis of acceptability including a combination of qualitative and quantitative measures to appropriately capture this construct (Sekhon, Cartwright, \& Francis, 2017). Despite the limitations, ensuring the post intervention measures were collected by a researcher not involved in the intervention was intended to maximise the likelihood of family members providing honest answers to the assessments and qualitative questions.

In summary, the changes in behaviour commonly observed in FTD often result in higher levels of family burden than seen in other dementias. PBS provides a potential approach to better support individuals with FTD and their family members living in the community. PBS was an acceptable intervention for the individuals with FTD and their families. Improvements were found for the levels of apathetic and disinhibited behaviours expressed by the person with dementia, and family members all reported a benefit from being involved in the program. A larger trial of 
implementing a PBS intervention with an FTD cohort will be an important next step in developing improved services tailored to the needs of this unique population.

\section{Acknowledgments}

The authors wish to thank the families who made this research possible.

\section{Funding details}

This work was supported in part by funding to ForeFront, a collaborative research group dedicated to the study of frontotemporal dementia and motor neuron disease, from the National Health and Medical Research Council (NHMRC) (GNT1037746) and the Australian Research Council (ARC) Centre of Excellence in Cognition and its Disorders (CE110001021). OP is supported by an NHMRC Senior Research Fellowship (GNT1103258).

\section{Disclosure of interest}

This work was supported in part by funding to ForeFront, a collaborative research group dedicated to the study of frontotemporal dementia and motor neuron disease, from the National Health and Medical Research Council (NHMRC) (GNT1037746) and the Australian Research Council (ARC) Centre of Excellence in Cognition and its Disorders (CE110001021). CMOC has no conflicts of interest to disclose. EM has no conflicts of interest to disclose. $\mathrm{CK}$ has no conflicts of interest to disclose. AF is supported by Flinders University, and Lifetime Support Authority Research Grant. $\mathrm{MH}$ has no conflicts of interest to disclose. OP is supported by an NHMRC Senior Research Fellowship (GNT1103258). 


\section{Data availability}

The data generated and analysed during the current study are available from the corresponding author on reasonable request. 


\section{References}

Algase, D. L., Beck, C., Kolanowski, A., Whall, A., Berent, S., Richards, K., \& Beattie, E. (1996). Need-driven dementia-compromised behavior: An alternative view of disruptive behavior. American Journal of Alzheimer's Disease \& Other Dementias, 11(6), 10-19. doi:10.1177/153331759601100603

Allen, D., James, W., Evans, J., Hawkins, S., \& Jenkins, R. (2005). Positive behavioural support: definition, current status and future directions. Tizard Learning Disability Review, 10(2), 4-11.

Andrews, G. J. (2006). Managing challenging behaviour in dementia: a person centred approach may reduce the use of physical and chemical restraints. $B M J$, 332: 741.

Armari, E., Jarmolowicz, A., \& Panegyres, P. K. (2013). The needs of patients with early onset dementia. American Journal of Alzheimer's Disease \& Other Dementias, 28(1), 42-46. doi:10.1177/1533317512466690

Bird, M., Llewellyn-Jones, R. H., Korten, A., \& Smithers, H. (2009). A controlled trial of a predominantly psychosocial approach to BPSD: treating causality. International Psychogeriatrics, 19(5), 874-891. doi:10.1017/S1041610206004790

Bird, M., Llewellyn-Jones, R. H., \& Korten, A. (2009). An evaluation of the effectiveness of a case-specific approach to challenging behaviour associated with dementia. Aging \& Mental Health, 13(1), 73-83. doi:10.1080/13607860802154499

Carr, E. G., Dunlap, G., Horner, R. H., Koegel, R. L., Turnbull, A. P., \& Sailor, W. (2002). Positive behavior support: evolution of an applied science. Journal of Positive Behavior Interventions, 4(1), 4-16. 
Cohen-Mansfield, J. (2000). Theoretical frameworks for behavioral problems in dementia. Alzheimer's Care Quarterly, 1(4), 8-21.

de Medeiros, K., Robert, P., Gauthier, S., Stella, F., Politis, A., Leoutsakos, J., . . . Lyketsos, C. (2010). The Neuropsychiatric Inventory-Clinician rating scale (NPI-C): Reliability and validity of a revised assessment of neuropsychiatric symptoms in dementia. International Psychogeriatrics, 22(6), 984-994. doi:10.1017/S1041610210000876

Durand, V. M., \& Crimmins, D. B. (1988). Identifying the variables maintaining selfinjurious behaviour. Journal of Autism and Developmental Disabilities, 18, 99-117.

Feeney, T. J., \& Achilich, J. (2014). Structured flexibility and context-sensitive behavioral support for the chronically cranky. NeuroRehabilitation, 34(4), 709-723. doi:10.3233/NRE-141088

Fisher, A., Bellon, M., Lawn, S., \& Lennon, S. (2019). Brain injury, behaviour support, and family involvement: putting the pieces together and looking forward. Disability and Rehabilitation. Online first 17 Jan 2019. doi: $10.1080 / 09638288.2018 .1522551$

Fisher, A., Bellon, M., Lawn, S., Lennon, S., \& Sohlberg, M. (2017). Family-directed approach to brain injury (FAB) model: a preliminary framework to guide family-directed intervention for individuals with brain injury, Disability and Rehabilitation, doi: 10.1080/09638288.2017.1407966

Gelinas, I., Gauthier, L., McIntyre, M., \& Gauthier, S. (1999). Development of a functional measure for persons with Alzheimer's disease: The Disability Assessment for Dementia. American Journal of Occupational Therapy, 53, 471-481. doi:10.5014/ajot.53.5.471 
Ghosh, A., Dutt, A., Bhargava, P., \& Snowden, J. (2013). Environmental dependency behaviours in frontotemporal dementia: have we been underrating them? Journal of Neurology, 260, 861-868.

Gitlin, L. N., Piersol, C. V., Hodgson, N., Marx, K., Roth, D. L., Johnston, D., . . . Lyketsos, C. G. (2016). Reducing neuropsychiatric symptoms in persons with dementia and associated burden in family caregivers using tailored activities: design and methods of a randomized clinical trial. Contemporary Clinical Trials, 49, 92-102. doi:10.1016/j.cct.2016.06.006

Gitlin, L. N., Winter, L., Burke, J., Chernett, N., Dennis, P. M., \& Hauck, W. W. (2008). Tailored activities to manage neuropsychiatric behaviors in persons with dementia and reduce caregiver burden: a randomized pilot study. American Journal of Geriatric Psychiatry, 16, 229-239.

Gorno-Tempini, M. L., Hillis, A. E., Weintraub, S., Kertesz, A., Mendez, M., Cappa, S. F., . . Grossman, M. (2011). Classification of primary progressive aphasia and its variants. Neurology, 76, 1006-1014. doi:10.1212/WNL.0b013e31821103e6

Haass, C., \& Neumann, M. (2016). Frontotemporal dementia: from molecular mechanisms to therapy. Journal of Neurochemistry, 138(Supp 1), 3-5.

Hassiotis, A., Robotham, D., Canagasabey, A., Romeo, R., Langridge, D., Blizard, R., ... King, M. (2009). Randomized, single-blind, controlled trial of a specialist behavior therapy team for challenging behavior in adults with intellectual disabilities. American Journal of Psychiatry, 166, 1278-1285.

Hodges, J. R. (2001). Frontotemporal dementia (Pick's disease): Clinical features and assessment. Neurology, 56(Supplement 4), S6-S10. 
Hodges, J. R., \& Piguet, O. (2018). Progress and challenges in frontotemporal dementia research: a 20-year review. Journal of Alzheimer's Disease, 62(Pt 6), $1-14$

Hornberger, M., Yew, B., Gilardoni, S., Mioshi, E., Gleichgerrcht, E., Manes, F., \& Hodges, J. R. (2014). Ventromedial-frontopolar prefrontal cortex atrophy correlates with insight loss in frontotemporal dementia and Alzheimer's disease. Human Brain Mapping, 35, 616-626.

Horner, R. H., Dunlap, G., Koegel, R. L., Carr, E. G., Sailor, W., Anderson, J., . . . O’Neill, R. E. (1990). Toward a technology of "nonaversive" behavioral support. Journal of the Association for Persons with Severe Handicaps, 15(3), $125-132$.

Kaiser, S., \& Panegyres, P. K. (2007). The psychosocial impact of young onset dementia on spouses. American Journal of Alzheimer's Disease \& Other Dementias, 21(6), 398-402. doi:10.1177/1533317506293259

Kazdin, A. E. (2016). Single-case experimental research designs. In A.E. Kazdin (Ed). Methodological issues and strategies in clinical research $\left(4^{\text {th }} \mathrm{ed}\right)$. Washington, DC: American Psychological Association.

Kortte, K. B., \& Rogalski, E. J. (2013). Behavioural interventions for enhancing life participation in behavioural variant frontotemporal dementia and primary progressive aphasia. International Review of Psychiatry, 25(2), 237-245.

LaVigna, G. W., \& Willis, T. J. (2005). A Positive Behavioural Support Model for Breaking the Barriers to Social and Community Inclusion. Tizard Learning Disability Review, 10(2), 16-23. 
LaVigna, G. W., \& Willis, T. J. (2012). The efficacy of positive behavioural support with the most challenging behaviour: the evidence and its implications. Journal of Intellectual and Developmental Disability, 37(3), 185-195.

Lawton, M. P., \& Brody, E. M. (1969). Assessment of older people: Self-maintaining and instrumental activities of daily living. Gerontologist, 9(3), 179-186.

Marczinski CA, Davidson W, Kertesz A. A longitudinal study of behavior in frontotemporal dementia and primary progressive aphasia. Cogn Behav Neurol 2004;17:185-190.

Martini de Oliveira, A., Radanovic, M., Homem de Mello, P. C., Buchain, P. C., Vizzotto, A. D., Harder, J......Forlenza, O. V. (2019). An intervention to reduce neuropsychiatric symptoms and caregiver burden in dementia: preliminary results from a randomized trial of the tailored activity programoutpatient version. International Journal of Geriatric Psychiatry, 34, 13011307

Massimo, L., Powers, C., Moore, P., Vesely, L., Avants, B., Gee, J., . . Grossman, M. (2009). Neuroanatomy of apathy and disinhibition in frontotemporal lobar degeneration. Dementia and Geriatric Cognitive Disorders, 27, 96-104. doi:10.1159/000194658

Massimo, L., Powers, J. P., Evans, L. K., McMillan, C. T., Rascovsky, K., Eslinger, P.....Grossman, M. (2015). Apathy in frontotemporal degeneration: neuroanatomical evidence of impaired goal-directed behavior. Frontiers in Human Neuroscience, 9:611.

McLean, B., \& Grey, I. (2012). A component analysis of positive behaviour support plans. Journal of Intellectual and Developmental Disability, 37(3), 221-231. 
Mendez, M. F., \& Shapira, J. S. (2011). Loss of emotional insight in behavioral variant frontotemporal dementia or "frontal anosodiaphoria". Consciousness and Cognition, 20, 1690-1696. doi: 10.1016/j.concog.2011.09.005

Mioshi, E., Foxe, D., Leslie, F., Savage, S., Hsieh, S., Miller, L., . . Piguet, O. (2013). The impact of dementia severity on caregiver burden in frontotemporal dementia and Alzheimer disease. Alzheimer Disease and Associated Disorders, 27(1), 68-73. doi:10.1097/WAD.0b013e318247a0bc

Mioshi, E., \& Hodges, J. R. (2009). Rate of change of functional abilities in frontotemporal dementia. Dementia and Geriatric Cognitive Disorders, 28, 419-426. doi:10.1159/000255652

Mioshi, E., Hsieh, S., Savage, S., Hornberger M., \& Hodges J. R. (2010). Clinical staging and disease progression in frontotemporal dementia. Neurology, 74(20), 1591-1597. doi: 10.1212/WNL.0b013e3181e04070

O'Connor, C. M., Clemson, L., da Silva, T. B. L., Piguet, O., Hodges, J. R., \& Mioshi, E. (2013). Enhancement of carer skills and patient function in the nonpharmacological management of frontotemporal dementia (FTD): a call for randomised controlled studies. Dementia \& Neuropsychologia, 7(2), 143-150.

O'Connor, C. M., Clemson, L., Hornberger, M., Leyton, C. E., Hodges, J. R., Piguet, O., \& Mioshi, E. (2016). Longitudinal change in everyday function and behavioral symptoms in frontotemporal dementia. Neurology: Clinical Practice, 6(5), 419-428. doi:10.1212/CPJ.0000000000000264

O’Connor, C. M., Clemson, L., Brodaty, H., Low, L. F., Jeon, Y. H., Gitlin, L. N., . . Mioshi, E. (2017a). The tailored activity program (TAP) to address behavioral disturbances in frontotemporal dementia: a feasibility and pilot study. Disability and Rehabilitation. doi:10.1080/09638288.2017.1387614 
O’Connor, C. M., Landin-Romero, R., Clemson, L., Kaizik, C., Daveson, N., Hodges, J. R., . . Mioshi, E. (2017b). Behavioral-variant frontotemporal dementia: distinct phenotypes with unique functional profiles. Neurology, 89, 570-577.

O’Neill, R. E., Horner, R. H., Albin, R. W., Sprague, J. R., Storey, K., \& Newton, J. S. (1997). Functional assessment and program development for problem behaviour: a practical handbook (2nd ed.). Pacific Grove, CA: Brooks/Cole. Rascovsky, K., Hodges, J. R., Knopman, D., mendez, M. F., Kramer, J. H., Neuhaus, J., . . Miller, B. L. (2011). Sensitivity of revised diagnostic criteria for the behavioural variant of frontotemporal dementia. Brain, 134, 2456-2477. doi:10.1093/brain/awr179

Ray-Subramanian, C. (2013). Motivation Assessment Scale. In F. R. Volkmar (Ed.), Encyclopedia of Autism Spectrum Disorders. New York, NY: Springer.

Rosen, H. J., Allison, S. C., Ogar, J. M., Amici, S., Rose, K., Dronkers, N., . . . Gorno-Tempini, M. L. (2006). Behavioural features in semantic dementia vs other forms of progressive aphasias. Neurology, 67, 1752-1756.

Savage, S. A., Piguet, O., \& Hodges, J. R. (2014). Giving words new life: generalization of word retraining outcomes in sematic dementia. Journal of Alzheimer's Disease, 40, 309-317.

Savage, S. A., Piguet, O., \& Hodges, J. R. (2015). “Knowing what you don't know”: language insight in semantic dementia. Journal of Alzheimer's Disease, 46, 187-198.

Sekhon, M., Cartwright, M., \& Francis, J. J. (2017). Acceptability of healthcare interventions: an overview of reviews and development of a theoretical framework. BMC Health Services Research, 17:88. doi:10.1186/s12913-017$2031-8$ 
Shinagawa, S., Ikeda, M., Fukuhara, R., \& Tanabe, H. (2006). Initial symptoms in frontotemporal dementia and semantic dementia compared with Alzheimer's disease. Dementia and Geriatric Cognitive Disorders, 21(2), 74-80. doi:10.1159/000090139

Shinagawa, S. (2013). Phenotypic variety in the presentation of frontotemporal dementia. International Review of Psychiatry, 25(2), 138-144.

Sinclair, C., Field, S., Williams, K., Blake, M., Bucks, R., Auret, K......Kurrle, S. (2018). Supporting decision-making: A guide for people living with dementia, family members and carers. Sydney: Cognitive Decline Partnership Centre.

Singh, N. N., Donatelli, L. S., Best, A., Williams, D. E., Barrera, F. J., Lenz, M. W., . . Moe, T. L. (1993). Factor structure of the Motivational Assessment Scale. Journal of Intellectual Disability Research, 37, 65-74.

Snowden, J. S., Bathgate, D., Varma, A., Blackshaw, A., Gibbons, Z. C., \& Neary, D. (2001). Distinct behavioural profiles in frontotemporal dementia and semantic dementia. Journal of Neurology, Neurosurgery and Psychiatry, 70, 323-332. doi:10.1136/jnnp.70.3.323

Staal, J. A., Amanda, S., Matheis, R., Collier, L., Calia, T., Hanif, H., \& Kofman, E. S. (2007). The Effects of Snoezelen (Multi-Sensory Behavior Therapy) and psychiatric care on agitation, apathy, and activities of daily living in dementia patients on a short term geriatric psychiatric inpatient unit. International Journal of Psychiatry in Medicine, 37(4), 357-370.

Tate, R. L., Perdices, M., McDonald, S., Togher, L., \& Rosenkoetter, U. (2014). The design, conduct and report of single-case research: resources to improve the quality of the neurorehabilitation literature. Neuropsychological Rehabilitation, 24, 315-331. doi: 10.1080/09602011.2013.875043 
Trombly, C. (1993). Anticipating the future: Assessment of occupational function. American Journal of Occupational Therapy, 47(3), 253-257.

Tsai, R. M., \& Boxer, A. L. (2014). Treatment of frontotemporal dementia. Current Treatment Options in Neurology, 16(11), 319. doi:10.1007/s11940-014-03190

Tsai, R. M., \& Boxer, A. L. (2016). Therapy and clinical trials in frontotemporal dementia: past, present, and future. Journal of Neurochemistry, 138(Supp 1), 211-221.

Van Langenhove, T., Leyton, C. E., Piguet, O., \& Hodges, J. R. (2016). Comparing longitudinal behavior changes in the primary progressive aphasias. Journal of Alzheimer's Disease. doi:10.3233/JAD-160010

Whitwell, J. L., Przybelski, S. A., Weigand, S. D., Ivnik, R. J., Vemuri, P., Gunter, J. L., . . Josephs, K. A. (2009). Distinct anatomical sutypes of the behavioural variant of frontotemporal dementia: a cluster analysis study. Brain, 132, 29322946.

Wicklund, A. H., Johnson, N., Rademaker, A., Weitner, B. B., \& Weintraub, S. (2007). Profiles of decline in activities of daily living in non-Alzheimer dementia. Alzheimer Disease and Associated Disorders, 21(1), 8-13. doi:10.1097/WAD.0b013e3180324549

Williams, G. B., Nestor, P. J., \& Hodges, J. R. (2005). Neural correlates of semantic and behavioural deficits in frontotemporal dementia. Neuroimage, 24, 10421051. doi:10.1016/j.neuroimage.2004.10.023

Zamboni, G., Huey, E. D., Krueger, F., Nichelli, P. F., Grafman, J. (2008). Apathy and disinhibition in frontotemporal dementia: insights into their neural correlates. Neurology, 71, 736-742. 
Table 1: Participant demographic characteristics at baseline

\begin{tabular}{ccccccccccc}
\hline Participant & Diagnosis & Sex & Age & $\begin{array}{c}\text { FRS } \\
\text { Rasch } \\
\text { score }\end{array}$ & $\begin{array}{c}\text { FRS } \\
\text { dementia } \\
\text { stage }\end{array}$ & $\begin{array}{c}\text { Disease } \\
\text { duration } \\
(\mathrm{yrs})\end{array}$ & $\begin{array}{c}\text { Education } \\
\text { (yrs) }\end{array}$ & $\begin{array}{c}\text { Family } \\
\text { member } \\
\text { Age }\end{array}$ & $\begin{array}{c}\text { Family } \\
\text { member } \\
\text { sex }\end{array}$ & $\begin{array}{c}\text { Family } \\
\text { member } \\
\text { education } \\
\text { (yrs) }\end{array}$ \\
\hline P1 & SD-left & M & 63.0 & 1.92 & Mild & 7.5 & 14.0 & 58.7 & F & 13.5 \\
P2 & SD-right & M & 70.0 & -1.03 & Severe & 6.5 & 20.0 & 62.5 & F & 17.0 \\
P3 & bvFTD & F & 66.3 & -0.59 & Severe & 4.9 & 12.0 & 72.5 & M & 15.0 \\
P4 & bvFTD & M & 65.5 & 0.16 & Moderate & 4.9 & 16.0 & 64.0 & F & 12.5 \\
\hline Mean & & & 66.19 & & & 5.92 & 15.5 & 64.43 & & 14.5 \\
\hline
\end{tabular}

bvFTD - behavioural variant frontotemporal dementia; FRS - Frontotemporal dementia Rating Scale; SD - semantic dementia 
Table 2: Motivational assessment scale outcomes for specifically identified apathetic and disinhibited behaviours for all study participants

\begin{tabular}{|c|c|c|c|c|c|c|}
\hline & Behaviours for each participant & $\begin{array}{c}\text { Behavioural } \\
\text { domain }\end{array}$ & Sensory & Escape & Attention & Tangible \\
\hline \multirow[t]{3}{*}{$\mathbf{P 1}$} & Shopping (spending money daily on "bargains") & $\mathrm{D}$ & 1 & 3 & 3 & 2 \\
\hline & Swearing (at wife and friends in public and at home) & $\mathrm{D}$ & 4 & 2 & 3 & 1 \\
\hline & Shoplifting & $\mathrm{D}$ & 1 & 2 & 2 & 2 \\
\hline \multirow[t]{3}{*}{$\mathbf{P 2}$} & Gambling & $\mathrm{D}$ & 1 & 2 & 2 & 2 \\
\hline & $\begin{array}{l}\text { Communication difficulties (impacting management e.g. not } \\
\text { responding to requests to change soiled clothes/bedding; in lieu of } \\
\text { verbal communication, pushes wife to access cooking) }\end{array}$ & $\mathrm{D}$ & 3 & 2 & 3 & 1 \\
\hline & $\begin{array}{l}\text { Shopping and shoplifting (e.g. grabbing items in shop; finding } \\
\text { "bargains"; taking cash from wife's wallet) }\end{array}$ & $\mathrm{D}$ & 1 & 2 & 3 & 2 \\
\hline \multirow[t]{3}{*}{$\mathbf{P 3}$} & Apathy (e.g. doing less around the house) & A & 1 & 2 & 3 & 4 \\
\hline & Decreased communication (e.g. not responding when addressed) & A & 2 & 1 & 2 & 3 \\
\hline & Anxiety (e.g. wants to leave restaurant as soon as finished eating) & $\mathrm{O}$ & 2 & 1 & 2 & 2 \\
\hline \multirow[t]{7}{*}{$\mathbf{P 4}$} & Changing plans last minute (e.g. not attend appointment) & $\mathrm{O}$ & 1 & 2 & 4 & 3 \\
\hline & Angry \& shouting (loud yelling heard by neighbours) & $\mathrm{D}$ & 3 & 2 & 4 & 1 \\
\hline & Apathy (not engaged in practical activities) & A & 1 & 2 & 2 & 2 \\
\hline & Rank of primary responses overall & & 1 & 3 & 4 & 2 \\
\hline & Rank of secondary responses overall & & 4 & 1 & 3 & 2 \\
\hline & Rank of apathetic primary responses & & 1 & 2 & & \\
\hline & Rank of disinhibited primary responses & & 1 & & & 2 \\
\hline
\end{tabular}

Scores are ratings $(1-4)$ derived from the MAS summed scores. A score of 1 indicated the most likely function of a behaviour.

Behavioural domain refers to whether the specifically identified behaviour relates to: D - Disinhibition, A - Apathy, or O - Other. 


\section{Figure:}

Figure 1a: Apathetic behaviours from baseline to post intervention for each individual participant.

Rated on the NPI-C apathetic domain scale. P - participant; bvFTD - behavioural variant frontotemporal dementia; $\mathrm{SD}$ - semantic variant frontotemporal dementia.

Figure 1b: Disinhibited behaviours from baseline to post intervention for each individual participant.

Rated on the NPI-C disinhibited domain scale. P - participant; bvFTD - behavioural variant frontotemporal dementia; SD - semantic variant frontotemporal dementia. 